\title{
Otolaryngology Open Access Journal
}

\section{Significance of Imaging in Head and Neck Cancer}

\section{Prakash VB*}

Private practitioner and Consultant Maxillofacial Radiologist, India

*Corresponding author: Prakash VB, MDS, Private practitioner and Consultant Maxillofacial Radiologist, Chennai, Tamilnadu, India, E-mail: drprakashvijayan@gmail.com

Abbreviations: CT: Computed Tomography; MR: Magnetic Resonance; DWI: Diffusion Weighted Imaging; BV: Blood Volume; BF: Blood Flow; MTT: Mean Transit Time; CP: Capillary Permeability; CBCT: Cone-Beam CT; GT: Gross Tumor Volume; PTV: Planning Target Volume

\section{Editorial}

Cross-sectional imaging examinations are fundamental to evaluate the head and neck region. Computed tomography (CT) scan, magnetic resonance (MR) imaging, and 18- $F$ fluorodeoxy glucose positron emission tomography (18-FFDG- PET) are presently the standard for initial workup and estimating treatment response in head \& neck cancer [1].

Ultrasonography is a useful imaging modality to detect soft tissue inflammatory changes and to evaluate the status of lymph nodes in head and neck cancer patients. It is an economical, noninvasive, easy-to-handle and costeffective diagnostic method. Lymph nodes changes evident on ultrasonographic evaluation is a useful factor in determining the metastatic nature of the cancer. USG examination performed is superior to clinical palpation in detecting cervical metastatic nodes [2].

Magnetic Resonance Imaging is advantageous over Computed Tomography as it provides a better soft-tissue differentiation. It is recommended in case of nasopharyngeal, base of tongue, sinonasal, or skull base carcinomas. Diffusion weighted imaging (DWI) is a noninvasive imaging biomarker to predict tumor response. DWI-MRuses water molecules motion in intracellular and extracellular space to reflect biological changes in tumor microenvironment. It plays a useful role in delineating soft-tissue changes due to radiation from recurrence [1].
CT has a high sensitivity (63-100\%) and moderate specificity (24-80\%) for differentiating recurrent tumor from post-treatment changes. It has upper hand in identifying areas of calcification and subtle bony cortical erosion. Deconvolution-based computed tomographic (CT) perfusion is a well-established imaging technique that can help assess physiologic parameters, such as blood volume (BV), blood flow (BF), mean transit time (MTT), and capillary permeability (CP) [3].

Computed tomography and Magnetic resonance imaging used for tumor staging enables detection of sub centimeter lesions [3]. Nodal spread is the most important factor that determines the prognosis in the management of patients. Ipsilateral or contralateral nodal metastasis reduces the 5-year disease-specific survival rate. The number of involved nodes, location, and evidence for extracapsular spread determine treatment and prognosis [2]. CT and MR imaging are the preferred modalities to image metastatic nodes. CT is superior to MR imaging in diagnosing the metastatic nodes in the head and neck. One of the hallmarks of the metastatic node is increase in size. On the basis of pathologic findings, a minimum axial diameter of $10 \mathrm{~mm}$ was the most effective size criterion, while the best size criterion was 1-2 mm greater for the nodes at levels IB and II. However, smaller ( $<10 \mathrm{~mm}$ ) nodes can harbor metastasis that could be imaged by metabolic imaging [2].

By using specific MRI techniques, functional information such as tumor perfusion vascular permeability, extracellular space tortuosity, metabolic status and hypoxia can also be obtained and used in radiotherapy of head and neck cancers [4].

Cone-beam CT (CBCT) is another novel form of 3dimensional imaging that can minimize patient 


\section{Otolaryngology Open Access Journal}

positioning inaccuracies. Images taken from a CBCT at the time of treatment can be overlaid on the original planning CT, and specialized software can be used to detect positioning errors with millimeter accuracy [5]. Similar to 2D portal imaging, two types of CBCT exist: MV and $\mathrm{kV}$. $\mathrm{CBCT}$ with $\mathrm{kV}$ imaging is reported to have better image contrast and smaller signal-to-noise ratios than MV CBCTs [6]. CBCT-based correction has also been used to increase treatment accuracy in the setting of IMRT, thus allowing for larger target doses, while simultaneously sparing healthy tissues [6].

The tonsillar fossa, the nasopharynx, the base of the tongue, and the pyrifo $\mathrm{rm}$ sinus are the common areas for occult primary in the case of head and neck squamous cell carcinoma. Fluoro Deoxy Glucose-Positron Emission Tomography has proved to be a complementary study for patients with unknown primary tumor of the head and neck when conventional evaluation fails [3]. In conventional CT scanning with or without contrast, delineating the gross tumor volume (GTV) is often difficult because there may not be a clear demarcation between tumor and normal tissue. PET-CT aids in GTV contouring. This is facilitated by the fact that the metabolic activity of the tumor clearly marks the boundary between active tumor and normal surrounding tissue. Accurate GTV definition with the assistance of PETCT facilitates the use of smaller expansions to create clinical target volumes and subsequent planning target volume (PTV), which later forms the basis for IMRT planning and creating optimal radiation-dose coverage of the tumor [3].

The FDG findings in post therapy examinations should be response therapy and time-specific. Increased FDG uptake will be seen in recently irradiated tissues and appears to be more intense in tissues that receive the highest radiation. Radiation-related FDG hypermetabolism may last 12-16 months after therapy [7].

There is discrepancy in the standard uptake volumes of radiation-related FDG uptake and tumor recurrence. This poses a problem for interpretation, if the PET/CT is performed within 2 months of radiation therapy. PET/CT is likely to be precise when performed at 3-4 months after the completion of radiation therapy. This is likely due to the fact that there is reduction in the nonspecific inflammatory uptake. The use of PET in addition to conventional staging in the staging of patients with newly diagnosed or recurrent carcinoma of the head and neck is considered: a) Safe

b) To increase accuracy for staging of regional lymph node metastases

c) To increase the detection of distant metastases, although these may occur infrequently

d) To detect additional lesions in approximately one-third of patients

e) To lead to changes in management in approximately $70 \%$ of patients when additional lesions are identified, most commonly changes in the radiotherapy regimen

f) Likely to lead to an improvement in overall patient outcomes when PET leads to an increase in the radiotherapy administered for the treatment of previously unsuspected local lymph node metastases.

Single Photon Emission Computed Tomography in head and neck SCC compares favorably with PET and is likewise useful in detecting occult tumors, especially naso- and oropharyngeal carcinomas and tumor recurrences (accuracy of $87 \%$ versus $64 \%$ for CT or MRI) [7]. The clinical and subclinical extension of head and neck SCC to the numerous bony structures of the face and skull base can be evaluated by bone SPECT, alone or associated with other scintigraphic techniques of direct tumor imaging [7]. Bone SPECT not only excludes bony invasion but reliably differentiates periosteal reaction from bone invasion by intraoral SCCs by semiquantitative analysis, allowing the surgeon to strip only the periosteum [7].

Imaging methods play an ever-expanding role in the management of head-and-neck cancer. Clinical examination allows direct visualization, whereas it cannot evaluate the deep extension of the disease. Diagnostic imaging in the anatomically complex head and neck region is performed for specific indications after thorough clinical examination. Conventional x-rays are easy to obtain but often cannot answer the clinical question and may yield confusing information leading to misdiagnosis. Therefore, neoplastic diseases of the head and neck are best evaluated with three dimensional techniques, optimally chosen to answer the specific clinical question, in accordance with current guidelines. Based on pertreatment imaging, individualized re-planning during radiotherapy may increase tumor control rates and thereby reduce the toxic effects on normal tissues. Although the oral cavity poses particular complexity in head and neck imaging, a sound understanding of radiological anatomy, common pathways of disease spread and current complementary technical approaches will improve detection and characterisation of cancer. 


\section{Otolaryngology Open Access Journal}

\section{References}

1. De Felice F, Daniela M, Vincenzo T (2015) Follow-Up in Head and Neck Cancer: A Management Dilemma. Advances in Otolaryngology 2015.

2. Prakash VB, Saraswathi GK, Harsha VBG (2017) Clinical evaluation and ultrasonographic characterization of Cervicofacial lymph nodes. American Journal of Oral Medicine and Radiology 4(1): 33-42.

3. Subramaniam RM, Truong M, Peller P, Sakai O, Mercier G (2010) Fluorodeoxyglucose-PositronEmission Tomography Imaging of Head and Neck Squamous Cell Cancer. AJNR Am J Neuroradiol 31(4): 598-604.
4. Metcalfe P, Liney GP, Holloway L, Walker A, Barton M, et al. (2013) The Potential for an Enhanced Role for MRI in Radiation-therapy Treatment Planning. Technol Cancer Res Treat 12(5): 429-446.

5. Sheedy SP, Welker KM, DeLone DR, Gilbertson JR (2006) CNS Metastases of Carcinoma Ex Pleomorphic Adenoma of the Parotid Gland. AJNR Am J Neuroradiol 27(7): 1483-1485.

6. Sameer NK, Daniel RS, Brent SR, Ajay PS (2009) Recent Advances in Image-Guided Radiotherapy for Head and Neck Carcinoma. Journal of Oncology 2009.

7. Chisin R (1999) Nuclear Medicine in Head and Neck Oncology: Reality and Perspectives. The journal of nuclear medicine 40(1): 91-95. 